\title{
Association of multimorbidity and changes in health-related quality of life following myocardial infarction: a UK multicentre longitudinal patient-reported outcomes study
}

T. Munyombwe ${ }^{1,2^{*}}$, T. B. Dondo ${ }^{1,2}$, S. Aktaa ${ }^{1,3}$, C. Wilkinson ${ }^{4}$, M. Hall ${ }^{1,2}$, B. Hurdus ${ }^{2,3}$, G. Oliver ${ }^{5}$, R. M. West ${ }^{6}$, A. S. Hall ${ }^{1,3}$ and C. P. Gale $e^{1,2}$

\begin{abstract}
Background: Multimorbidity is prevalent for people with myocardial infarction (MI), yet previous studies investigated single-health conditions in isolation. We identified patterns of multimorbidity in MI survivors and their associations with changes in HRQOL.

Methods: In this national longitudinal cohort study, we analysed data from 9566 admissions with MI from 77 National Health Service hospitals in England between 2011 and 2015. HRQoL was measured using EuroQol 5 dimension (EQ5D) instrument and visual analogue scale (EQVAS) at hospitalisation, 6, and 12 months following MI. Latent class analysis (LCA) of pre-existing long-term health conditions at baseline was used to identify clusters of multimorbidity and associations with changes in HRQoL quantified using mixed effects regression analysis.
\end{abstract}

Results: Of 9566 admissions with MI (mean age of 64.1 years [SD 11.9], 7154 [75\%] men), over half (5119 [53.5\%] had multimorbidities. LCA identified 3 multimorbidity clusters which were severe multimorbidity (591; 6.5\%) with low HRQoL at baseline (EQVAS 59.39 and EQ5D 0.62) which did not improve significantly at 6 months (EQVAS 59.92, EQ5D 0.60); moderate multimorbidity (4301; 47.6\%) with medium HRQoL at baseline (EQVAS 63.08, EQ5D 0.71) and who improved at 6 months (EQVAS 71.38, EQ5D 0.76); and mild multimorbidity (4147, 45.9\%) at baseline (EQVAS 64.57, EQ5D 0.75) and improved at 6 months (EQVAS 76.39, EQ5D 0.82). Patients in the severe and moderate groups were more likely to be older, women, and presented with NSTEMI. Compared with the mild group, increased multimorbidity was associated with lower EQ-VAS scores (adjusted coefficient: -5.12 [95\% Cl -7.04 to -3.19$]$ and $-0.98[-1.93$ to -0.04$]$ for severe and moderate multimorbidity, respectively.

The severe class was more likely than the mild class to report problems in mobility, OR 9.62 (95\% confidence interval: 6.44 to 14.36), self-care 7.87 (4.78 to 12.97), activities 2.41 (1.79 to 3.26), pain 2.04 (1.50 to 2.77), and anxiety/ depression 1.97 (1.42 to 2.74 ).

\footnotetext{
* Correspondence: T.munyombwe@leeds.ac.uk

'Leeds Institute of Cardiovascular and Metabolic Medicine, University of Leeds, Leeds LS2 9JT, UK

${ }^{2}$ Leeds Institute for Data Analytics, University of Leeds, Leeds, UK

Full list of author information is available at the end of the article
}

(c) The Author(s). 2021 Open Access This article is licensed under a Creative Commons Attribution 4.0 International License, which permits use, sharing, adaptation, distribution and reproduction in any medium or format, as long as you give appropriate credit to the original author(s) and the source, provide a link to the Creative Commons licence, and indicate if changes were made. The images or other third party material in this article are included in the article's Creative Commons licence, unless indicated otherwise in a credit line to the material. If material is not included in the article's Creative Commons licence and your intended use is not permitted by statutory regulation or exceeds the permitted use, you will need to obtain permission directly from the copyright holder. To view a copy of this licence, visit http://creativecommons.org/licenses/by/4.0/ The Creative Commons Public Domain Dedication waiver (http://creativecommons.org/publicdomain/zero/1.0/) applies to the data made available in this article, unless otherwise stated in a credit line to the data. 
Conclusions: Among Ml survivors, multimorbidity clustered into three distinct patterns and was inversely associated with HRQoL. The identified multimorbidity patterns and HRQoL domains that are mostly affected may help to identify patients at risk of poor HRQoL for which clinical interventions could be beneficial to improve the HRQOL of MI survivors.

Trial registration: ClinicalTrials.gov NCT01808027 and NCT01819103

Keywords: Multimorbidity, Health-related quality of life, EQ5D, Myocardial infarction,

\section{Background}

Multimorbidity, the presence of two or more long-term health conditions in an individual, is a major clinical and public health challenge [1, 2]. Around two thirds of patients with cardiovascular diseases have at least one long-term health condition [3]. Co-morbidities are present in $60 \%$ of patients hospitalised with myocardial infarction (MI) and are associated with significant years of life lost [4]. Following MI, patients frequently report poor health related quality of life (HRQoL), which may not recover [5], and is independently associated with higher rates of death at 1 year [6, 7]. Poor HRQoL is more commonly reported among women [8,9], older people with frailty $[5,10]$, non-white ethnicity [11], and in those that experience bleeding complications relating to dual anti-platelet therapy [12]. Several previous studies have looked at individual chronic health conditions and their impact on health-related quality of life in MI survivors. Patients with long-term health conditions such as hypertension [13], diabetes [14], angina [15], depression [16], and chronic obstructive pulmonary disease (COPD) report poor HRQoL. Specific disease combinations may have greater effects than others on functional, physical and mental status [17], quality of life [18], and mortality $[4,19]$.

However, there is a paucity of longitudinal studies that have investigated the association of multimorbidity and temporal changes in HRQoL [20-22]. Existing studies are limited by a focus on mortality as the primary end point $[4,21]$, use of cross-sectional designs [20,23], and focusing on individual disease in isolation. Different disease combinations may have greater effects than others on patient outcomes. A previous study [4] found an association of combinations of disease clusters with patient survival in MI patients. We have extended this research by looking at how combinations of disease clusters in survivors of MI impact on changes in HRQoL. Notably, there are few large-scale datasets available that combine clinical data with robust evaluation of temporal changes in HRQoL for patients with MI. In this study, we identified baseline multimorbidity patterns, compare changes in HRQoL across multimorbidity clusters, and investigate the associations of these clusters with changes in HRQoL after MI.

\section{Methods \\ Data and participants \\ Setting}

We analysed data from Evaluation of the Methods and Management of Acute Coronary Events, EMMACE-3 and EMMACE-4, which are multicentre longitudinal national cohort studies of outcomes following MI combining survey data with national clinical registration data (ClinicalTrials.gov NCT01808027 and NCT01819103) [24]. The study included patients aged 18 years and over who were hospitalized with MI, defined by the third universal definition as either ST-elevation myocardial infarction (STEMI) or non-STEMI (NSTEMI) [25]. Participants were recruited from 77 National Health Service (NHS) hospitals in England between November 1, 2011, and June 24, 2015. They were consented to participate by a trained researcher during their hospital admission (data flow is shown in Additional file 1: Figure S1).

Patients at a terminal stage of any illness, and those for whom follow-up would be inappropriate or impractical, were excluded. Consenting patients were asked to complete a self-administered questionnaire at the time of enrolment in hospital, and at 1, 6, and 12 months following discharge from hospital. This included information about HRQoL assessed using the three level EuroQol 5-dimension (EQ-5D-3L) instrument [24]. For non-responders who were alive and who had not withdrawn from the study, repeat questionnaires were sent by post on up to three occasions before the date of the next follow-up contact. Data for consenting patients were linked to the national clinical register of MI admissions in the UK (Myocardial Ischaemia National Audit Project, MINAP [26]) to gather information about patients medical history including presence of hypertension, diabetes mellitus, angina, asthma or chronic obstructive pulmonary disease (COPD), cerebrovascular disease (CVSD), peripheral vascular disease (PVD), chronic heart failure, chronic renal failure, type of MI (NSTEMI or STEMI), and in-hospital as well as postdischarge treatments and medications.

\section{Health-related quality of life}

The outcome of this study was HRQoL assessed using self-reported EQ-5D-3L [27]. This contains two 
subscales: a descriptive system (EQ-5D) and a visual analogue scale (EQ-VAS). EQ-5D comprises five dimensions: mobility, self-care, usual activities, pain/discomfort, and anxiety/depression. Each domain has three levels (3L): no problems, some problems, and extreme problems. The EQ-5D-3L dimensions data may be summarised as a single index score ranging from -0.5 to 1 , with scores less than 0 indicating states 'worse than death', 0 indicating no quality of life, or 'death', and 1 indicating full health and therefore no problems in any domain. The index score was standardised to the UK population [28]. The EQ-VAS score ranges from 0 to 100 , with 0 denoting the worst and 100 the best, health state imaginable. The EQ-5D questionnaire has previously been validated in patients following MI [29]. A difference in score of 7 for VAS and 0.05 for EQ-5D score are regarded as clinically important [30], and these thresholds were used to define a clinically important change between subgroups.

\section{Exposure}

The exposure was multimorbidity clusters based on 7 pre-existing long-term health conditions recorded in the MINAP registry including hypertension, diabetes mellitus, asthma or chronic obstructive pulmonary disease (COPD), cerebrovascular disease (CVSD), peripheral vascular disease (PVD), chronic heart failure, and chronic renal failure. In the data source used for the study, the Myocardial Ischaemia National Audit Project (MINAP) [26] registry information on multi-morbidities is given as binary variables. No further detail is given beyond this; therefore, we were restricted to use the information as recorded.

\section{Other variables}

Sociodemographic, health characteristics, and clinical variables included age, sex, ethnicity (white vs other), smoking status (never vs ex or current), body mass index (BMI) $\left(\mathrm{kg} / \mathrm{m}^{2}\right)$, past medical history of MI, angina, diagnosis (STEMI or NSTEMI), revascularisation (percutaneous coronary intervention $[\mathrm{PCI}]$ vs. no PCI; coronary artery bypass graft $[C A B G]$ surgery vs no CABG surgery), medications (aspirin, $\beta$ blockers, statins, and ACE inhibitors), and referral for cardiac rehabilitation (yes/no).

\section{Statistical analysis}

Patient characteristics according to multimorbidity clusters were described using frequencies and percentages for categorical data and for continuous data as means and standard deviation. Chi-square test and ANOVA were used to assess univariate associations between categorical and continuous patients' characteristics and multimorbidity clusters, respectively. We corrected for multiple testing in the tables using the Hochberg correction, using a false discovery rate of 0.05 .

Latent class analysis (LCA) [31] using Mplus software version 8 was used to identify clusters of multimorbidity for 7 pre-existing long-term health conditions recorded in the MINAP registry at hospital admission including hypertension, diabetes mellitus, asthma or chronic obstructive pulmonary disease (COPD), cerebrovascular disease (CVSD), peripheral vascular disease (PVD), chronic heart failure, and chronic renal failure.

Latent class analysis (LCA) is a statistical technique used to determine subgroups within populations that share certain outward characteristics [31]. In LCA, class membership is based on probability of belonging to a class membership given the pattern of responses they have on indicator variables, and there are no clear cut assignments. LCA is a "person-centred" approach of deriving typologies, unlike "variable-centred" tradition that uses arbitrary cut-offs for classifying individual cases [32]. We used LCA instead of cluster analysis [33] because unlike cluster analysis or k-means clustering, LCA is model-based and an evaluation of how well a proposed LCA model represents the data can be conducted [33] using Bayesian information criterion (BIC) [34], Akaike's information criteria (AIC) [35], and Bootstrap likelihood ratio test (BLRT) [36] $p$ values. We fitted several latent class models varying the number of classes up to 5 classes to identify the best class solution with 1000 random starting values each with 100 iterations. Bootstrap $p$ values based on 500 replications were used to assess model fit. The model goodness of fit statistics, entropy, classification matrix, class frequencies, and class conditional probabilities is reported in Additional file 1: Table S1, Table S2, and Table S3. The utility of using LCA has been demonstrated by other researchers who used it to identify clinical phenotypes with differential treatment responses [37] and patient outcomes [4, 38, 39].

The optimal LCA model was selected based on Bayesian information criterion (BIC), Akaike's information criteria (AIC), Bootstrap likelihood ratio test (BLRT) $p$ values, and clinical interpretation. For the BIC and AIC the optimal model is the model with the smallest value, and for the BLRT, the optimal number of clusters is where the $p$ value becomes non-significant at significance level 0.05. A three-class multimorbidity solution provided the best latent class model fit based on BIC, AIC, and BLRT $p$ values. Patient allocation was based on posterior probabilities of belonging to a class. In order to determine the adjusted association of baseline patient characteristics with multimorbidity cluster membership, we fitted multinomial logistic regression models and reported using odds ratios and their corresponding 95\% confidence intervals. 
Multilevel linear regression analysis [40] of longitudinal changes in EQ-VAS scores (in hospital, 1 month, 6 months, and 12 months) was performed to investigate the associations of multimorbidity clusters and temporal changes in patient perceptions of health. The outcomes data are repeated measurements overtime, and patients are clustered within hospitals; therefore, data are not independent, and a multilevel linear model was used to account for the clustering in the data. The multilevel models were fitted in steps, first an unconditional means model was used to determine the significance of the 2 random-effect terms (hospital and patient). To check whether the linear model was appropriate, we examined the distribution of residuals to check that there were approximately normally distributed. The normal probability plots are reported in Additional file 1: Figure S2. Where the normality assumption was violated, the multilevel Tobit regression [41] models were fitted and compared to the multilevel linear model results and the results were similar. Tobit regression models are commonly used to analyse patient reported outcome measures data with ceiling and floor effects.

The analysis adjusted for time (categorised as baseline, 1 month, 6 months, and 12 months), age, sex, ethnicity (white vs other), smoking status (never vs ex or current), past medical history of MI, angina, diagnosis (STEMI or NSTEMI), revascularisation (percutaneous coronary intervention $[\mathrm{PCI}]$ vs. no $\mathrm{PCI}$; coronary artery bypass graft [CABG] surgery vs no CABG surgery), medications (aspirin, $\beta$ blockers, statins, and ACE inhibitors), referral for cardiac rehabilitation (yes/no), and interactions of time and multimorbidity class. The confounders were selected based on clinical consideration and previous research $[4,42]$.

Multilevel linear regression analysis of longitudinal changes in EQ-5D scores (in hospital, 1 month, 6 months, and 12 months) was performed to investigate the associations of multimorbidity clusters and temporal changes in HRQoL. Effect sizes (regression coefficients) and their corresponding 95\% confidence intervals were used to assess the adjusted magnitude of the difference in EQ-VAS, EQ-5D scores across multimorbidity classes.

To investigate the association of multimorbidity clusters and changes in HRQoL measured by EQ-5D dimensions, five multilevel logistic regression models were fitted for the EQ-5D dimensions (mobility, self-care, activities, pain, and anxiety, and depression) adjusting for age, sex, ethnicity (white versus other) smoking status (never vs ex or current), past medical history of MI, angina, diagnosis (STEMI or NSTEMI), revascularisation (percutaneous coronary intervention [PCI] vs. no PCI; coronary artery bypass graft [CABG] surgery vs no CABG surgery), medications (beta-blockers, statins, angiotensin converting enzymes (ACE), aspirin), cardiac rehabilitation (yes/no), and interactions of time and multimorbidity.

The 'extreme problem' category for some domains of the EQ5D measure was endorsed by few individuals for some domains (e.g., self-care and mobility); therefore, we combined the EQ-5D levels 'some problems' and 'extreme problems' and the responses were binary (no problems vs some/extreme problems) and adjusted odds ratios (OR) and their corresponding 95\% confidence intervals were used to assess the adjusted associations of multimorbidity classes and EQ-5D dimensions.

\section{Missing data}

In longitudinal studies, missing data are commonly encountered, subjects can be missed at a particular assessment time; therefore, subjects may provide outcome data at some, but not all study time points resulting in incomplete data. Participants might drop out of the study or could be lost to follow up. In this study, there was missing outcome data over time. A sensitivity analysis was conducted comparing baseline characteristics of patients with complete data and those that dropped out (Additional file 1: Table S4). The drop outs were not significantly different from the followed up subjects in sex, ethnicity, previous angina, chronic renal failure, and PVD but were significantly different in age, diagnosis (NSTEMI, STEMI), Index of Multiple Deprivation (IMD), smoking status, history of previous acute MI (AMI), PCI, prevalence of diabetes, baseline EQ-5D, and EQ-VAS scores. In order to include patients with incomplete outcome data and to mitigate against biases which may arise as a result of such an omission, we used a multilevel model that includes all participants even if they were not assessed at all 4 time points.

All statistical tests were two-sided, and statistical significance was considered at $p<0.05$. Analyses were conducted using stata (IC) version 15

\section{Patient involvement}

Whilst no patients were involved in setting the research question or the study design, we have co-produced this research manuscript with a patient with prior MI who provided input into the interpretation of the research findings, gave a critical review of the manuscript, and will work with our research team in ensuring its widespread dissemination.

\section{Results}

\section{Patient characteristics}

Descriptive statistics for the cohort are shown in Table 1. From 16,780 acute coronary syndrome hospitalisations across the 77 recruiting hospitals in England between 2010 and 2015, we excluded 4250 who did not have a diagnosis of MI and 2964 non-index hospitalisations, 
Table 1 Patient characteristics by multimorbidity class

\begin{tabular}{|c|c|c|c|c|c|}
\hline Variable & $\begin{array}{l}\text { Total } \\
\text { cohort } \\
n=9566\end{array}$ & $\begin{array}{l}\text { Multimorbidity class } 1 \\
\text { (severe) } 591 \text { (6.5\%) }\end{array}$ & $\begin{array}{l}\text { Multimorbidity class } 2 \\
\text { (moderate) } 4301(47.6 \%)\end{array}$ & $\begin{array}{l}\text { Multimorbidity class } 3 \text { (mild) } \\
4147(45.9 \%)\end{array}$ & $P$ value $^{\dagger}$ \\
\hline Age, mean (SD) & $\begin{array}{l}64.1 \\
(11.9)\end{array}$ & $74.8(9.3)$ & $68.8(10.3)$ & $57.7(10.5)$ & $<0.001^{*}$ \\
\hline Women, $n$ (\%) & $\begin{array}{l}2384 \\
(24.9)\end{array}$ & $177(30.0)$ & $1274(29.7)$ & $803(19.4)$ & $<0.001^{*}$ \\
\hline IMD, mean (SD) & $\begin{array}{l}23.0 \\
(15.7)\end{array}$ & $24.0(16.2)$ & $22.3(15.2)$ & $23.7(16.1)$ & 0.04 \\
\hline BMI, mean(SD) & $28.7(6.0)$ & $29.8(5.4)$ & $28.9(5.5)$ & $28.2(6.5)$ & $<0.001^{*}$ \\
\hline $\begin{array}{l}\text { Ethnicity (Caucasian), } n \\
(\%)\end{array}$ & $\begin{array}{l}8136 \\
(85.1)\end{array}$ & $507(94.8)$ & $3639(94.2)$ & 3530 (93.3) & 0.41 \\
\hline $\begin{array}{l}\text { Ex/current smoking } \\
\text { status, } n(\%)\end{array}$ & $\begin{array}{l}6248 \\
(65.3)\end{array}$ & $368(64.6)$ & $2709(64.2)$ & 5979 (67.2) & $<0.001^{*}$ \\
\hline NSTEMI, $n$ (\%) & $\begin{array}{l}5658 \\
(59.2)\end{array}$ & $508(86.0)$ & $2870(66.7)$ & $2046(49.3)$ & $<0.001^{*}$ \\
\hline STEMI, $n$ (\%) & $\begin{array}{l}3908 \\
(40.8)\end{array}$ & $83(14.0)$ & 1431 (33.3) & $2101(50.7)$ & \\
\hline \multicolumn{6}{|l|}{ Co-morbidities } \\
\hline Diabetes, n (\%) & $\begin{array}{l}1714 \\
(17.9)\end{array}$ & $375(64.7)$ & $1143(27.1)$ & $107(2.6)$ & $<0.001^{*}$ \\
\hline $\begin{array}{l}\text { Peripheral vascular } \\
\text { disease, } n(\%)\end{array}$ & 317 (3.3) & $121(20.5)$ & $168(3.9)$ & $24(0.6)$ & $<0.001^{*}$ \\
\hline $\begin{array}{l}\text { Cerebrovascular } \\
\text { disease, } n(\%)\end{array}$ & $428(4.5)$ & $122(20.6)$ & $297(6.9)$ & 0 & $<0.001^{*}$ \\
\hline $\begin{array}{l}\text { Asthma or COPD, } n \\
\text { (\%) }\end{array}$ & $\begin{array}{l}1166 \\
(12.2)\end{array}$ & $147(24.9)$ & $651(15.1)$ & 358 (8.6) & $<0.001^{*}$ \\
\hline $\begin{array}{l}\text { Chronic renal failure, } n \\
(\%)\end{array}$ & $289(3.0)$ & $235(39.8)$ & $45(1.1)$ & $8(0.2)$ & $<0.001^{*}$ \\
\hline Heart failure, $n(\%)$ & $212(2.2)$ & $203(34.4)$ & 0 & $6(0.1)$ & $<0.001^{*}$ \\
\hline Hypertension, $n(\%)$ & $\begin{array}{l}4078 \\
(42.6)\end{array}$ & $408(69.0)$ & $3295(76.6)$ & $337(8.1)$ & $<0.001^{*}$ \\
\hline Previous MI, n (\%) & $\begin{array}{l}1522 \\
(15.9)\end{array}$ & $384(65.0)$ & $1006(23.4)$ & $114(2.8)$ & $<0.001^{*}$ \\
\hline Angina, $n(\%)$ & $\begin{array}{l}1792 \\
(18.7)\end{array}$ & $374(63.3)$ & $1322(30.7)$ & $80(1.9)$ & $<0.001^{*}$ \\
\hline Previous PCI, $n$ (\%) & $899(9.4)$ & $169(28.7)$ & $617(14.4)$ & $98(2.4)$ & $<0.001^{*}$ \\
\hline CABG surgery, $n$ (\%) & $643(6.7)$ & $152(25.8)$ & $452(10.5)$ & $29(0.7)$ & $<0.001^{*}$ \\
\hline \multicolumn{6}{|l|}{$\begin{array}{l}\text { Discharge } \\
\text { medications }\end{array}$} \\
\hline Aspirin, $n(\%)$ & $\begin{array}{l}8147 \\
(85.2)\end{array}$ & $464(79.2)$ & $3626(84.7)$ & 3636 (87.9) & $<0.001^{*}$ \\
\hline ACE inhibitors, $n$ (\%) & $\begin{array}{l}7609 \\
(79.5)\end{array}$ & $418(71.3)$ & $3389(79.2)$ & $3436(83.1)$ & $<0.001^{*}$ \\
\hline Beta-blockers, n (\%) & $\begin{array}{l}7592 \\
(79.7)\end{array}$ & $430(73.4)$ & $3340(78.0)$ & 3439 (83.1) & $<0.001^{*}$ \\
\hline Statin, $n(\%)$ & $\begin{array}{l}8140 \\
(85.1)\end{array}$ & $477(81.4)$ & 3619 (84.6) & 3636 (87.9) & $<0.001^{*}$ \\
\hline \multicolumn{6}{|l|}{ Rehabilitation } \\
\hline $\begin{array}{l}\text { Cardiac rehabilitation, } \\
n(\%)\end{array}$ & $\begin{array}{l}8509 \\
(88.9)\end{array}$ & $502(85.1)$ & $3854(90.3)$ & 3877 (94.5) & $<0.001^{*}$ \\
\hline
\end{tabular}

IMD Index of Multiple Deprivation, BMI body mass index, ACE angiotensin-converting enzyme, STEMI ST-elevation myocardial infarction, NSTEMI non-STEMI, CABG coronary artery bypass grafting, $P C l$ percutaneous coronary intervention, $A M l$ acute myocardial infarction, $C O P D$ Chronic Obstructive Pulmonary Disease. ${ }^{\dagger}$ Obtained from chi-squared or analysis of variance (ANOVA) as appropriate. *Significant after Hochberg correction using a false discovery rate of 0.05 
leaving an analytical cohort of 9566 patients (3908 STEMI, 5658 NSTEMI, Additional file 1: Figure S1 in the supplement). The EQ-5D-3L questionnaire response rates were 97.5\% (9332/9566), 74.7\% (6679/8945), 63.9\% (5572/ $8719)$, and $62.7 \%(5047 / 8043)$ at hospitalisation, 1 month, 6 months, and 12 months, respectively. Reasons for nonparticipation at each stage included death and withdrawals from the study. Sixty-nine out of 9566 patients died in hospital. There were significant differences in some baseline characteristics between the patients who dropped out and those who completed the study (Additional file 1: Table S4).

Baseline demographic data were missing in less than $5 \%$ of cases, except for the Index of Multiple Deprivation (IMD) (55.0\%) and ethnicity (9.3\%). Overall, 25.1\% $(2,397)$ of the analytical cohort were women. The mean age was 64.1 (standard deviation [SD] 11.9) years; mean body mass index (BMI) $28.7(6.04) \mathrm{kg} / \mathrm{m}^{2}$, median IMD 18.5 (interquartile range [IQR] 10.9 to 31.8), and 6248 $(65.3 \%)$ were smokers or ex-smokers.

\section{Long-term health conditions in MI patients}

Frequently observed long-term health conditions included hypertension 4078 (42.6\%), angina 1792 (18.7\%), PVD 428 (4.5\%), diabetes mellitus 1714 (17.9\%), COPD 1166 (12.2\%), CVSD 428 (4.5\%), chronic renal failure 289 (3.0\%), and heart failure 212 (2.2\%). Overall, 53.5\% of participants (5119) had one or more long-term health conditions; 3562 out of $9566(37.2 \%)$ had none; 3157 (33.0\%) had one, 1414 (14.8\%) had two, 419 (4.4\%) had three, $110(1.2 \%)$ had 4, and $19(0.2 \%)$ had five or more co-morbidities. Co-morbidity data were not recorded in $885(9.3 \%)$ cases. Co-morbidities were more prevalent in women $(1442,60.1 \%)$ than men $(3674,51.3 \%)$.

\section{Multimorbidity clusters in MI patients}

LCA identified three distinct multimorbidity classes and were clinically labelled as class 1: severe multimorbidity, 591 (6.5\%), class 2: moderate multimorbidity, 4301 (47.6\%), and class 3: mild multimorbidity, 4147 (45.9\%).

The majority of health conditions were more prevalent in the severe class: diabetes (severe 64.7\%; moderate 27.1\%; and mild 2.6\%), PVD (20.5\%, 3.9\% and $0.6 \%$ ), CVSD $(20.6 \%, 6.9 \%$ and $0 \%), \operatorname{CRF}(39.8 \%, 1.1 \%$ and $0.2 \%$ ) and heart failure (34.4\%, $0.0 \%$ and $0.1 \%$ ), and asthma or COPD $(24.9 \%, 15.1 \%$ and $8.6 \%)$; however, hypertension was more prevalent in the moderate class (severe $69 \%$ moderate $76.6 \%$ and mild $8.1 \%$ ) (Fig. 1).

\section{Factors associated with multimorbidity class membership} Compared with the mild multimorbidity class, patients in the severe and moderate multimorbidity classes were older (mean age of $74.8,68.8$ vs 57.5 years), had a higher proportion of women $(30.0 \%, 29.7 \%$ vs $19.4 \%)$, more commonly presented with NSTEMI $(86.0 \%, 66.7 \%$ vs $49.3 \%)$ and more often had a history of angina (63.3\%, $30.7 \%$ vs $1.9 \%$ ), previous MI ( $65.0 \%, 23.4 \%$ vs $2.8 \%$ ), CABG $(25.8 \%, 10.5 \%$ vs $0.7 \%)$, and previous PCI $(28.7 \%$, $14.4 \%$ vs $2.4 \%$ ). Compared to the severe class, the mild and moderate classes were more likely to have STEMI (50.7\% and $33.3 \%$ vs. $14.0 \%$ ) (Table 1 ).

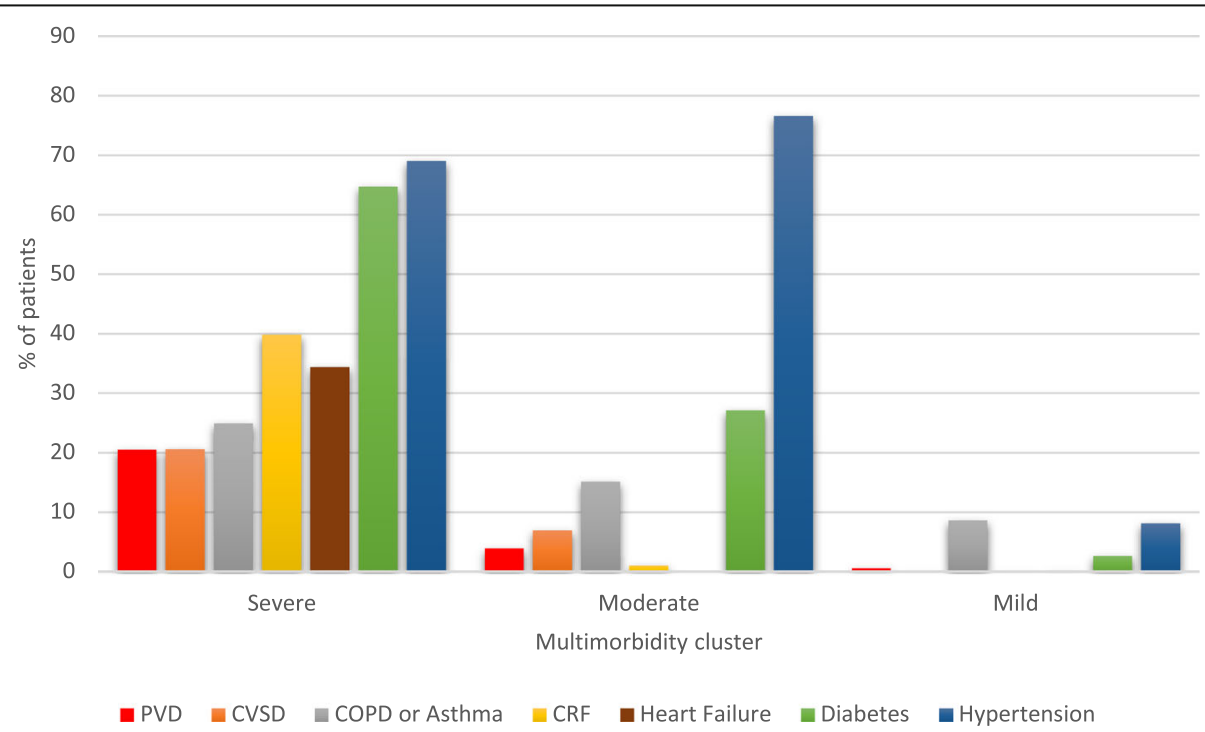

Fig. 1 Percent of patients with conditions in each multimorbidity class. Severe multimorbidity patients tended to have high levels of all comorbidities, moderate multimorbidity patients tended to have hypertension, and diabetes, and mild multimorbidity were patients with few comorbidities. Note COPD indicates chronic obstructive pulmonary disease; CVSD, cerebrovascular disease; PVD, peripheral vascular disease; CRF, chronic renal failure 
Multimorbidity and perceptions of health state

Table 2 shows descriptive statistics for EQ-VAS scores by multimorbidity clusters. Without adjustment for differences in patient characteristics, perceptions of health state measured by EQ-VAS scores were worse in the severe multimorbidity class compared to the mild and moderate classes suggesting better recovery of HRQoL in the mild and moderate multimorbidity classes compared to the severe class and these differences persisted at 12 months (Fig. 2). Compared with the moderate and mild classes, patients in the severe class had lower EQVAS scores at hospitalisation (59.39 vs. 63.08, 64.57), at 30 days (58.95 vs $68.20,71.73), 6$ months (59.92 vs $71.38,76.39)$, and 12 months (61.73 vs $72.05,77.69)$.

Table 2 EQ-VAS and EQ-5D utility scores by follow-up time and multimorbidity class

\begin{tabular}{|c|c|c|c|c|}
\hline Variable & $\begin{array}{l}\text { Multimorbidity class } 1 \text { (severe) } \\
591(6.5 \%)\end{array}$ & $\begin{array}{l}\text { Multimorbidity class } 2 \text { (moderate) } \\
4301(47.6 \%)\end{array}$ & $\begin{array}{l}\text { Multimorbidity class } 3 \text { (mild) } \\
4147(45.9 \%)\end{array}$ & $P$ value \\
\hline \multicolumn{5}{|l|}{ EQ-5D, mean (SD) } \\
\hline Baseline & $0.62(0.31)$ & $0.71(0.29)$ & $0.75(0.28)$ & $<0.001^{*}$ \\
\hline 1 month & $0.61(0.30)$ & $0.73(0.26)$ & $0.78(0.24)$ & $<0.001^{*}$ \\
\hline 6 months & $0.60(0.30)$ & $0.75(0.26)$ & $0.82(0.24)$ & $<0.001^{*}$ \\
\hline 12 months & $0.59(0.30)$ & $0.76(0.27)$ & $0.83(0.24)$ & $<0.001^{*}$ \\
\hline \multicolumn{5}{|l|}{$\begin{array}{l}\text { Utilities: EQ-VAS, } \\
\text { mean (SD) }\end{array}$} \\
\hline Baseline & $59.39(20.83)$ & $63.08(20.30)$ & $64.57(21.01)$ & $<0.001^{*}$ \\
\hline 1 month & $58.95(19.36)$ & $68.20(18.27)$ & $71.73(17.73$ & $<0.001^{*}$ \\
\hline 6 months & $59.92(20.79)$ & $71.38(18.54)$ & 76.39 (17.22) & $<0.001^{*}$ \\
\hline 12 months & $61.73(19.43)$ & $72.05(18.60)$ & $77.69(17.31)$ & $0.001^{*}$ \\
\hline \multicolumn{5}{|l|}{$\begin{array}{l}\text { EQ-5D dimensions } \\
\text { baseline }\end{array}$} \\
\hline Mobility & $398(67.34)$ & $1833(42.62)$ & $1048(25.27)$ & $<0.001^{*}$ \\
\hline Self-care & $163(27.58)$ & $690(16.04)$ & $399(9.62)$ & $<0.001^{*}$ \\
\hline Activities & $370(62.61)$ & $2115(49.17)$ & $1837(44.30)$ & $<0.001^{*}$ \\
\hline Pain & $288(48.73)$ & $1662(38.64)$ & $1178(28.41)$ & $<0.001^{*}$ \\
\hline Anxiety and depression & $221(37.39)$ & $1473(34.25)$ & $1381(33.30)$ & 0.081 \\
\hline \multicolumn{5}{|l|}{ EQ-5D 30 days } \\
\hline Mobility & $264(71.35)$ & $1364(43.86)$ & $683(24.03)$ & $<0.001^{*}$ \\
\hline Self-care & $120(32.61)$ & $481(15.49)$ & $215(7.58)$ & $<0.001^{*}$ \\
\hline Activities & $275(74.53)$ & $1862(60.06)$ & $1498(53.05)$ & $<0.001^{*}$ \\
\hline Pain & $228(61.79)$ & $1484(47.81)$ & 1077 (38.08) & $<0.001^{*}$ \\
\hline Anxiety and depression & $160(43.72)$ & $1152(37.13)$ & $1066(37.54)$ & 0.05 \\
\hline \multicolumn{5}{|l|}{ EQ-5D 6 months } \\
\hline Mobility & 209 (70.13) & $1149(43.31)$ & $538(23.08)$ & $<0.001^{*}$ \\
\hline Self-care & 97 (33.33) & $387(14.63)$ & $173(7.42)$ & $<0.001^{*}$ \\
\hline Activities & $216(71.76)$ & $1220(46.02)$ & 766 (32.90) & $<0.001^{*}$ \\
\hline Pain & $208(69.57)$ & $1280(48.25)$ & $808(34.71)$ & $<0.001^{*}$ \\
\hline Anxiety and depression & $129(43.14)$ & $821(30.99)$ & $730(31.32)$ & $<0.001^{*}$ \\
\hline \multicolumn{5}{|l|}{ EQ-5D 12 months } \\
\hline Mobility & $207(76.10$ & $1080(44.68)$ & $477(22.60)$ & $<0.001^{*}$ \\
\hline Self-care & $97(35.27)$ & $389(16.11)$ & $166(7.88)$ & $<0.001^{*}$ \\
\hline Activities & $203(74.09)$ & $1091(45.19)$ & $571(27.05)$ & $<0.001^{*}$ \\
\hline Pain & $195(70.91)$ & $1126(46.94)$ & $673(32.08)$ & $<0.001^{*}$ \\
\hline Anxiety and depression & $99(36.00)$ & $696(28.88)$ & $568(26.92)$ & $0.006^{*}$ \\
\hline
\end{tabular}

Values are given as the mean (standard deviation) for EQ-VAS, EQ-5D utility scores. Values are given as frequencies (percentages) for EQ-5D dimensions. *Significant after Hochberg correction using a false discovery rate of 0.05 


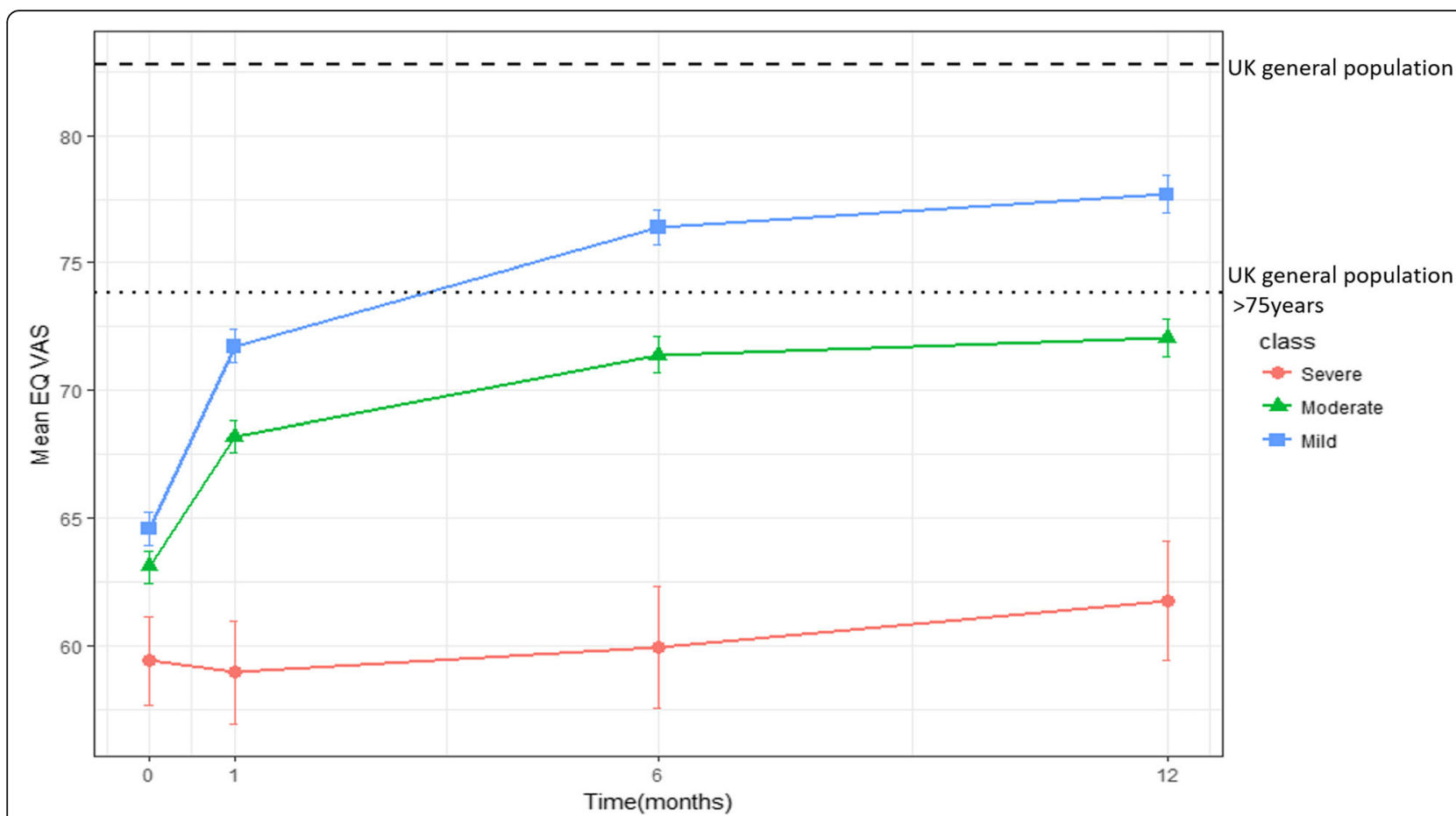

Fig. 2 Temporal changes of HRQoL by multimorbidity class. The EQ-VAS scores range from 0 (worst) to 100 (best) health status with a difference of 7 points considered clinically meaningful. Patients with severe multimorbidity have worse health-related quality of life, shown by EQ-VAS scores, at baseline and through 12 months of follow-up

\section{Multimorbidity and HRQoL measured by EQ-5D}

Responses from the EQ-5D dimensions showed that during hospitalisation, $69.1 \%(6607 / 9566)$ of participants reported $\geq$ one problem across the EQ-5D dimensions, which increased to $73.9 \%(4935 / 6679)$ at 1 month, decreased to $62.6 \%(3491 / 5572)$ at 6 months, and $59.7 \%(3011 / 5047)$ at 12 months. Overall, the most frequently reported problems at baseline were for activities $(50.1 \%)$, followed by mobility (37.6\%), pain (35.5\%), anxiety and depression (35.4\%), and self-care (14.4\%). At 12 months, they were pain $(41.8 \%)$, followed by activities (38.8\%), mobility (36.7\%), anxiety and depression (28.6\%), and self-care (13.6\%). Table 2 shows descriptive statistics for EQ-5D scores by multimorbidity clusters.

Compared with the mild class, the percentage reporting $\geq 1$ problem on the EQ-5D dimension at hospitalisation was higher in the severe and moderate classes (severe $85.3 \%$; moderate $72.8 \%$ vs mild $65.5 \%$ ), at 1 month $(89.2 \%, 76.1 \%$ vs $69.0 \%), 6$ months $(85.9 \%, 67.5 \%$ vs $53.9 \%)$, and 12 months (88.6\%, $65.3 \%$ vs $49.2 \%)$. At hospitalisation, individuals in the severe class were more likely to report having problems in all EQ-5D dimensions compared to the moderate and mild classes: mobility (severe $67.3 \%$ vs. mild $42.6 \%$, mild $25.3 \%$, respectively), self-care $(27.6 \%$ vs $16.0 \%, 9.6 \%)$, activities (62.6\% vs $49.2 \%, 44.3 \%)$, pain $(48.7 \% \%$ vs. $38.6 \%, 28.4 \%)$, anxiety and depression (37.4\% vs $34.3 \%, 33.3 \%$ ), and the prevalence of problems continued to be higher in the severe class at 1,6 , and 12 months. Individuals in the severe class were also likely to report problems with mobility, self-care, activities, pain, anxiety, and depression at 1, 6, and 12 months (Table 2). Compared with the moderate and mild classes, patients in the severe class had lower EQ-5D scores at hospitalisation (0.62 vs. $0.71,0.75)$, at 30 days $(0.61$ vs $0.73,0.78), 6$ months $(0.60$ vs $0.75,0.82)$, and 12 months $(0.59$ vs $0.76,0.83)$.

\section{Adjusted results from multilevel modelling of longitudinal EQ-VAS, EQ-5D scores, and multimorbidity classes}

Compared with the baseline, the adjusted average health state scores improved at 1 month (difference 4.62, 95\% CI 4.04 to 5.19 ) and 6 months $(3.43,2.11$ to 4.75$)$, but declined at 12 months $(-1.10,-3.55$ to 1.35$)$ suggesting a poorer health state at 12 months and greatest improvement at one month (Table 3). After adjusting for covariates, compared to the mild multimorbidity class, increased multimorbidity was associated with a significant decline in EQ-VAS scores (adjusted coefficient: $-5.12[95 \% \mathrm{CI}-7.04$ to -3.19$]$ and -0.98 [-1.93 to -0.04 ] for severe and moderate multimorbidity, respectively. Similarly, increased multimorbidity was associated with a decline in EQ-5D scores (adjusted coefficient: -0.16 [95\% CI -0.18 to -0.13$]$ and -0.05 [ -0.06 to 
Table 3 Adjusted parameter estimates from multilevel modelling of EQ-VAS scores and multi-morbidity classes, regression coefficient, and 95\% confidence intervals

\begin{tabular}{|c|c|c|}
\hline Variable & $\begin{array}{l}\text { Regression coefficient } \\
(95 \% \mathrm{Cl})\end{array}$ & $P$ value \\
\hline Intercept & 48.39 (40.06 to 56.72$)$ & \\
\hline \multicolumn{3}{|l|}{ Month, baseline (ref) } \\
\hline 1 month & 4.62 (4.04 to 5.19) & $<0.001^{*}$ \\
\hline 6 months & 3.43 (2.11 to 4.75$)$ & $<0.001^{*}$ \\
\hline 12 months & $-1.10(-3.55$ to 1.35$)$ & 0.38 \\
\hline \multicolumn{3}{|l|}{$\begin{array}{l}\text { Multimorbidity classes } \\
\text { Mild (ref) }\end{array}$} \\
\hline Moderate & $-0.98(-1.93$ to -0.04$)$ & 0.04 \\
\hline Severe & $-5.12(-7.04$ to -3.19$)$ & $<0.001^{*}$ \\
\hline \multicolumn{3}{|l|}{ Diagnosis(STEMI) ref } \\
\hline Diagnosis (NSTEMI) & $-0.26(-1.19$ to 0.65$)$ & 0.57 \\
\hline Age & 0.12 (0.08 to 0.15$)$ & $<0.001^{*}$ \\
\hline Women & $-4.17(-5.02$ to -3.32$)$ & $<0.001^{*}$ \\
\hline Ethnicity White & $0.81(-1.61$ to 3.24 & 0.51 \\
\hline Ex/current smoking status & $-1.07(-1.84$ to -0.30$)$ & 0.01 \\
\hline Previous Ml & $-1.37(-2.64$ to -0.10$)$ & 0.03 \\
\hline Previous Angina & $-2.15(-3.27$ to -1.04$)$ & $<0.001^{*}$ \\
\hline \multicolumn{3}{|l|}{ Treatments } \\
\hline Previous $\mathrm{PCl}$ & $-1.53(-2.96$ to 0.11$)$ & 0.035 \\
\hline Previous CABG surgery & $-3.03(-4.64$ to -1.42$)$ & $<0.001^{*}$ \\
\hline
\end{tabular}

Adjusting for age, sex, ethnicity (white versus other) smoking status (never vs ex or current), past medical history of MI, angina, diagnosis (STEMI or NSTEMI), revascularisation (percutaneous coronary intervention $[\mathrm{PCl}]$ vs. no $\mathrm{PCl}$; coronary artery bypass graft [CABG] surgery vs no CABG surgery), medications (B-blockers, statins, ACE, aspirin), cardiac rehabilitation (yes/no), and interactions of time and multimorbidity. Note: $C A B G$ coronary artery bypass grafting, $P C l$ percutaneous coronary intervention, $M l$ myocardial infarction, STEMI ST-elevation myocardial infarction, NSTEMI non-ST-elevation myocardial infarction, "Significant after Hochberg correction using a false discovery rate of 0.05

-0.04] for severe and moderate multimorbidity classes, respectively (Additional file 1: Table S5).

\section{Adjusted results from multilevel modelling of longitudinal EQ-5D dimensions and multimorbidity classes}

The multilevel logistic regression analysis results for the association of multimorbidity classes with five EQ-5D dimensions are shown in Fig. 3. After adjustment, individuals in the severe class were more likely than those in the mild class to report problems in mobility, OR 9.62 (95\% confidence interval: 6.44 to 14.36 ), self-care 7.87 (4.78 to 12.97 ), activities 2.41 (1.79 to 3.26 ), pain 2.04 (1.50 to 2.77), and anxiety/depression 1.97 (1.42 to 2.74).

Similarly, individuals in the moderate class were more likely than those in the mild class to report problems with their mobility: 2.21 (1.82 to 2.69), self-care 2.05 (1.56 to 2.69), activities 1.10 (0.95 to 1.27 ), pain 1.33 (1.14 to 1.55 ), and anxiety/depression 1.26 (1.07 to 1.48 ).

\section{Discussion}

In this nationwide study of 9566 patients with MI who were followed for 1 year, multimorbidity was common and associated with low HRQoL. Uniquely, our study identifies three distinct clusters of multimorbidity: severe (older/multiple chronic conditions), moderate (older/ hypertension/diabetes), and mild (younger/low rates of chronic conditions). Different patterns of multimorbidity were associated with different HRQoL trajectories. We found an inverse relationship between extent of multimorbidity and improvement in HRQoL over time. Those with a high burden of multimorbidity at baseline had poor HRQoL, which failed to recover following MI. Notably, the EQ-VAS scores reported in patients with MI across all three multimorbidity classes were lower than those which have been reported in other common chronic conditions such as COPD [43] and heart failure [44], and UK general population [45]. Severe baseline multimorbidity was adversely associated with the EQ-5D dimensions mobility, self-care, activities, pain, anxiety, and depression. Indeed, severe multimorbidity was associated with an average decrease of 5 points in EQ-VAS scores compared to the mild multimorbidity cluster. Older MI survivors with high rates of multimorbidity, and patients with hypertension and diabetes are more likely to be associated with poor HRQoL.

Several previous studies have looked at the associations of individual chronic health conditions and healthrelated quality of life but a few focussed on combinations of disease clusters and their impact on healthrelated quality of life in MI survivors. Our study made a contribution to existing research by investigating the impact of different multimorbidity clusters on changes in HRQoL in MI survivors. Understanding disease clusters and their impact on HRQoL provides more insight for designing patient-centred care interventions. Our findings are consistent with previous studies that have found a decline in health-related quality of life in patients with hypertension, diabetes [46-48], and lung diseases $[14,19]$. Such chronic conditions are related with stress, and their co-existence may accentuate the impact on psychological wellbeing owing to care demands [23]. Similar to other previous studies, we found a significant association between increased multimorbidity and anxiety and depression. This finding is consistent with previous research that reported an association between depression and cardiovascular diseases $[49,50]$. Whilst it is known that a greater burden of comorbidities is associated with increased depression and other mental health conditions [1], the complex interplay between these remains unclear [50].

Our findings have similarities and differences from another study [51] that found five distinct multimorbidity patterns in Asian patients with heart failure. 


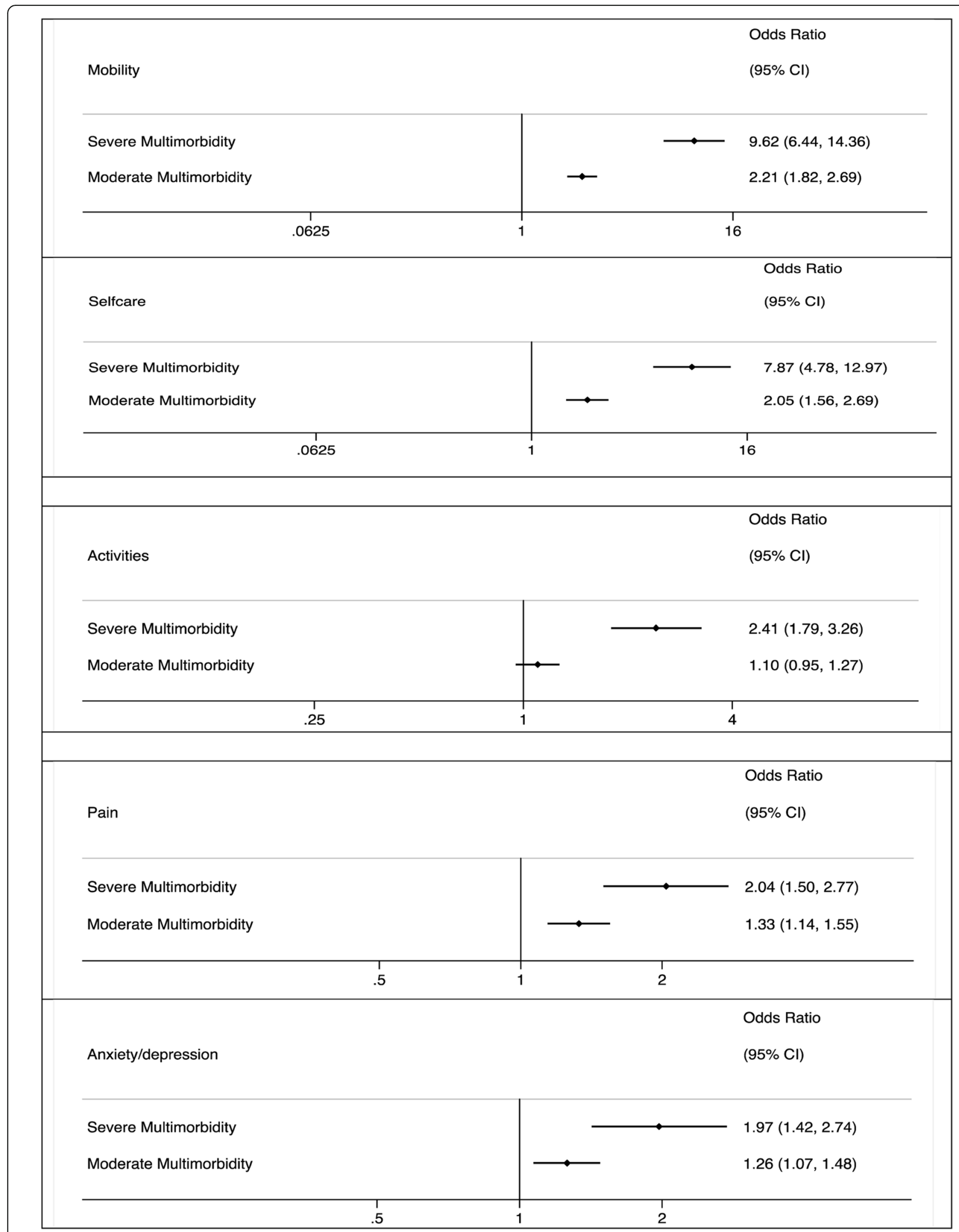

Fig. 3 (See legend on next page.) 
(See figure on previous page.)

Fig. 3 Association of multimorbidity classes and EQ-5D dimensions, (Mobility, Self-care, Activities, Pain and Anxiety/depression), odds ratios and 95\% confidence intervals (reference group, mild multimorbidity). Adjusting for age, sex, ethnicity (white versus other) smoking status (never vs ex or current), past medical history of $\mathrm{Ml}$, angina, diagnosis (STEMI or NSTEMI), revascularisation (percutaneous coronary intervention [PCI] vs. no PCl; coronary artery bypass graft [CABG] surgery vs no CABG surgery), cardiac rehabilitation (yes/no) and interactions of time and multimorbidity, and medications

Discrepancies in number of classes between our study and that by Tromp et al. could be due to differences in the cohort health conditions. The study by Tromp et al. used a cohort of patients with heart failure, whilst our cohort were patients with myocardial infarction. Furthermore, we classified patients using 7 health conditions whilst the study by Tromp et al. used more than 7 health conditions including those that were not available in our study such as coronary artery disease, previous stroke, chronic kidney disease, peptic ulcer, cancer, liver disease, and dementia. Similar to our study, Tromp et al. identified a class with a high probability of having hypertension and diabetes and a class with low comorbidity rates.

Similar to our findings a UK study [4] using the same 7 health conditions that we used in our study found three distinct multimorbidity patterns in patients with myocardial infarction. There are similarities and differences in comorbidities combinations between our clusters and those found by Hall et al. The three multimorbidity patterns reported by Hall et al. were elderly with high multimorbidity (tended to have heart failure, PVD, and hypertension), medium multimorbidity patterns (tended to have PVD and hypertension), and younger with low multimorbidity (patients with few comorbidities). We also found an elderly high multimorbidity cluster with high comorbidities and a younger, low multimorbidity cluster with few comorbidities. However, the multimorbidity patterns were different between our medium multimorbidity cluster and that reported by Hall et al. The medium multimorbidity cluster reported in our study tended to have diabetes and hypertension whilst that reported by Hall et al. tended to have PVD and hypertension. These discrepancies could be due to different cohorts used by the two studies. Hall's study used the MINAP registry which is much bigger ( $n=$ $693,388)$ and more representative of the target population than the EMMACE cohort $(n=9566)$ which comprises of a select cohort of patients who volunteered to participate in the study hence may exhibit selection bias. Furthermore, the EMMACE study excluded patients at a terminal stage of any illness, and those for whom follow-up was inappropriate or impractical. The study by Hall et al. had older patients with mean age 70.7 years compared to 64 years for the EMMACE study. Latent class analysis applied to a larger cohort may yield a different number of classes with different patterns [52]; therefore, there is need for more research to externally validate our study findings.

\section{Strengths and limitations}

To our knowledge, this is the first study to map the distribution of different co-morbidity patterns among patients with MI and determine their impact on temporal changes in HRQoL using a large nationwide longitudinal cohort study. The breadth of the data increases generalisability, but with sufficient depth to allow multi-level modelling, and latent class analysis. We have extended previous knowledge by identifying distinct multimorbidity clusters and their impact on HRQoL in MI survivors. The EQ-5D captures important aspects of health status that may be affected by an MI, including mobility, activities, self-care, depression or anxiety, and pain. Whilst we recognise that using a disease-specific metric may have added additional insights, use of a generic tool allowed us to interpret the findings in the context of a range of other clinical conditions. Limitations of this work include the potential for participation bias and survivorship bias, in common with all such studies. In this study, multimorbidity classes were determined using seven health conditions that were recorded in the MINAP registry, more research using more health conditions is needed and to investigate whether the multimorbidity clusters change over time using latent transition models.

\section{Clinical implications}

The identification of multimorbidity patterns and their impact on changes in HRQoL, and EQ-5D domains (mobility, activities, pain, anxiety, and depression) may help inform the design of targeted interventions [23], whereby therapies that better control co-morbidities in MI survivors may translate into meaningful improvement in HRQoL. Furthermore, patients with very high levels of multimorbidity might benefit from an early multidisciplinary team involvement to directly improve specific domains of their HRQoL. Multidisciplinary efforts to effectively manage cardiovascular risk factors (particularly diabetes and hypertension) may have an additional secondary preventive role.

\section{Conclusion}

This multi-centre longitudinal study of over 9500 survivors of hospitalised MI found that multimorbidity was adversely associated with dimensions of HRQoL including mobility, self-care, and activities of daily living, pain, anxiety, and depression. Moreover, a data-driven 
approach has enabled the identification of three clinically distinct multimorbidity clusters with significant differences in HRQoL and who may be suitable for tailored interventions to improve and maintain their HRQoL following MI. Older survivors of MI with high rates of multimorbidity, and patients with hypertension and diabetes are more likely to be associated with poor HRQoL. As such, we recommend specific interventions to target these subgroups to improve their HRQoL.

\section{Abbreviations}

HRQoL: Health-related quality of life; COPD: Chronic obstructive pulmonary disease; EMMACE: Evaluation of the Methods and Management of Acute Coronary Events; MI: Myocardial infarction; STEMI: ST-elevation myocardial infarction; NSTEMI: Non-STEMI; NHS: National Health Service; EQ-5D3L: EuroQol 5-dimension 3-levels; MINAP: Myocardial Ischaemia National Audit Project; CVSD: Cerebrovascular disease; PVD: Peripheral vascular disease; EQ-VAS: Visual analogue scale; PCI: Percutaneous coronary intervention; CABG: Coronary artery bypass graft; B-Blockers: Beta-blockers; ACE: Angiotensin-converting enzyme; IMD: Index of Multiple Deprivations

\section{Supplementary Information}

The online version contains supplementary material available at https://doi. org/10.1186/s12916-021-02098-y.

Additional file 1. Association of multimorbidity and changes in health related quality of life following myocardial infarction: A UK multicentre longitudinal patient-reported outcomes study. Figure S1-[Number of patients who enrolled in EMMACE 3 and 4]. Figure S2-[Normal probability plots for level 1 and level 2 residuals for (A) EQVAS and (B) EQ5D models]. Table S1-[latent class analysis model selection goodness of fit statistics]. Table S2-[Proportions of latent class based on their most likely latent class membership]. Table S3-[Class conditional probabilities of responses to the 7 comorbidities]. Table S4-[Comparison of baseline characteristics between respondent and non-respondents at 12 months]. Table S5-[Adjusted parameter estimates from multilevel modelling of EQ-5D scores and multi-morbidity classes, regression coefficient and 95\% confidence intervals].

\section{Acknowledgements}

We gratefully acknowledge the contributions from all hospitals, healthcare professionals, patients, and researchers who participated in the Evaluation of the Methods and Management of Acute Coronary Events studies.

\section{Authors' contributions}

TM and TBD were responsible for the literature search. CPG and ASH contributed to the conception of the research, funding acquisition, project administration, supervision, study design, and data collection and provided expert clinical advice in the interpretation of the results and were involved in manuscript writing. TM was involved in funding acquisition, formal data analysis, writing the original draft, and revising and editing. TBD was involved in data analysis and writing of the manuscript. RW provided statistical expert advice and was involved in funding acquisition and manuscript writing. $\mathrm{MH}$ was involved in funding acquisition, interpretation of the data, and manuscript writing. SA, BH, and CW were involved in interpretation of data and manuscript writing. GO was involved as a patient advisor in the interpretation of the research and the writing of the manuscript. CPG and TM are the guarantors for this study. All authors made critical revisions and provided intellectual content to the manuscript, approved the final version to be published, and agreed to be accountable for all aspects of the work. TBD and BH verified the data. The authors read and approved the final manuscript.

\section{Funding}

This research was funded by the National Institute for Health Research (NIHR/CS/009/004) and BHF Project Grant no. PG/19/54/34511. CPG was funded by the National Institute for Health Research (NIHR/CS/009/004). TBD was funded by the British Heart Foundation (PG/19/54/34511). MH was funded by the Wellcome Trust (206470/Z/17/Z). CW is funded by the NIHR as an academic clinical lecturer.

\section{Availability of data and materials}

The PIs of the EMMACE cohort datasets that were used in this study are not able to share individual level data due to ethical reasons. Additional related documents can be requested through the corresponding author of this manuscript.

\section{Declarations}

Ethics approval and consent to participate

Ethics approval EMMACE-3 and EMMACE-4 were given a favourable ethical opinion by the Leeds (West), and West Midlands Research Ethics committees (REC reference: 10/H131374 and 12/WM/0431) are registered on ClinicalTrials. gov (NCT01808027 and NCT01819103) and were adopted onto the National Institute for Health Research Comprehensive Research Network portfolio (9102). Patients were consented to participate into the EMMACE studies by a trained researcher during their hospital admission.

\section{Consent for publication}

Not applicable

\section{Competing interests}

Prof Gale reports grants from Abbot Diabetes, personal fees from Amgen, personal fees from AstraZeneca, personal fees from Bayer, grants from BMS, personal fees from Daiichy Sankyo, and personal fees from Vifor Phamra outside the submitted work. Dr Wilkinson reports a research grant from BMS.

The other authors declare that they have no competing interests.

\section{Author details}

'Leeds Institute of Cardiovascular and Metabolic Medicine, University of Leeds, Leeds LS2 9JT, UK. ${ }^{2}$ Leeds Institute for Data Analytics, University of Leeds, Leeds, UK. ${ }^{3}$ Department of Cardiology, Leeds Teaching Hospitals NHS Trust, Leeds, UK. ${ }^{4}$ Population Health Sciences Institute, Faculty of Medical Sciences, Newcastle University, Newcastle upon Tyne, UK. ${ }^{5}$ Lancashire, UK.

${ }^{6}$ Leeds Institute of Health Sciences, University of Leeds, Leeds, UK.

Received: 21 April 2021 Accepted: 16 August 2021

Published online: 28 September 2021

\section{References}

1. Barnett K, Mercer SW, Norbury M, Watt G, Wyke S, Guthrie B. Epidemiology of multimorbidity and implications for health care, research, and medical education: a cross-sectional study. Lancet. 2012;380(9836):37-43. https://doi. org/10.1016/S0140-6736(12)60240-2.

2. Sciences AM. Multimorbidity: a priority for global health research. London: Academy of Medical Sciences; 2018.

3. Rashid M, Kwok CS, Gale CP, Doherty P, Olier I, Sperrin M, et al. Impact of co-morbid burden on mortality in patients with coronary heart disease, heart failure, and cerebrovascular accident: a systematic review and metaanalysis. Eur Heart J. 2016;3(1):20-36.

4. Hall M, Dondo TB, Yan AT, Mamas MA, Timmis AD, Deanfield JE, et al. Multimorbidity and survival for patients with acute myocardial infarction in England and Wales: latent class analysis of a nationwide population-based cohort. PLoS Med. 2018;15(3):e1002501. https://doi.org/10.1371/journal. pmed. 1002501.

5. Munyombwe T, Hall M, Dondo TB, Alabas OA, Gerard O, West RM, et al. Quality of life trajectories in survivors of acute myocardial infarction: a national longitudinal study. Heart. 2019.

6. Pocock S, Bueno H, Licour M, Medina J, Zhang L, Annemans L, et al. Predictors of one-year mortality at hospital discharge after acute coronary syndromes: a new risk score from the EPICOR (longtErm follow uP of antithrombotic management patterns In acute CORonary syndrome patients) study. Eur Heart J. 2015;4(6):509-17. https://doi.org/10.1177/204 8872614554198

7. Sajobi T, Wang M, Santana M, Southern D, Liang Z, Galbraith D, et al. Trajectories of health-related quality of life in coronary artery disease. Circulation. 2018;11(3) 
8. Dreyer RP, Xu X, Liu S, Ding Q, Krumholz HM, Zheng X, et al. Sex differences in health outcomes at one year following acute myocardial infarction: a report from the China Patient-Centered Evaluative Assessment of Cardiac Events prospective acute myocardial infarction study. Eur Heart J. 2019;8(3): 273-82. https://doi.org/10.1177/2048872618803726.

9. Huffman MD, Baldridge AS, Zhao L, Lloyd-Jones DM, Mohanan PP, Devarajan $\mathrm{R}$, et al. Health-related quality of life at 30 days among indian patients with acute myocardial infarction: results from the ACS QUIK trial. Circulation. 2019;12(2).

10. Beska B, Coakley D, MacGowan G, Adams-Hall J, Wilkinson C, Kunadian V. Frailty and quality of life after invasive management for non-ST elevation acute coronary syndrome. Heart. 2021.

11. Webster RA, Thompson DR, Larkin D, Mayou RA, Martin CR. Quality of life in a mixed ethnic populationafter myocardial infarction. Eur J Pers Cent Healthc. 2017:5(3):295-9.

12. Amin AP, Wang TY, McCoy L, Bach RG, Effron MB, Peterson ED, et al. Impact of bleeding on quality of life in patients on DAPT: insights from TRANSLATE-ACS. J Am Coll Cardiol. 2016;67(1):59-65. https://doi.org/10.10 6/j.jacc.2015.10.034.

13. Warraich HJ, Peterson ED, Wang TY, Kaltenbach LA, Fonarow GC. Adverse change in employment status after acute myocardial infarction: analysis from the TRANSLATE-ACS study. Circulation. 2018;11(6).

14. Peña-Longobardo $L$, Rodríguez-Sánchez B, Mata-Cases M, Rodríguez-Mañas L, Capel M, Oliva-Moreno J. Is quality of life different between diabetic and non-diabetic people? The importance of cardiovascular risks. PLoS One. 2017;12(12):e0189505. https://doi.org/10.1371/journal.pone.0189505.

15. Fanaroff AC, Kaltenbach LA, Peterson ED, Hess CN, Cohen DJ, Fonarow GC, et al. Management of persistent angina after myocardial infarction treated with percutaneous coronary intervention: insights from the TRANSLATE-ACS study. J Am Heart Assoc. 2017;6(10):e007007.

16. Kim JM, Stewart R, Bae KY, Kang HJ, Kim SW, Shin IS, et al. Effects of depression co-morbidity and treatment on quality of life in patients with acute coronary syndrome: the Korean depression in ACS (K-DEPACS) and the escitalopram for depression in ACS (EsDEPACS) study. Psychol Med. 2015;45(8):1641-52. https://doi.org/10.1017/S003329171400275X.

17. Salisbury C, Man M-S, Bower P, Guthrie B, Chaplin K, Gaunt DM, et al. Management of multimorbidity using a patient-centred care model: a pragmatic cluster-randomised trial of the 3D approach. Lancet. 2018; 392(10141):41-50. https://doi.org/10.1016/S0140-6736(18)31308-4

18. N'Goran AA, Déruaz-Luyet A, Haller DM, Zeller A, Rosemann T, Streit $S$, et al. Comparing the self-perceived quality of life of multimorbid patients and the general population using the EQ-5D-3L. PLoS One. 2017;12(12):e0188499. https://doi.org/10.1371/journal.pone.0188499.

19. Lewis EF, Pfeffer MA, Solomon SD, Li Y, Weinfurt KP, Velazquez EJ, et al. Impact of cardiovascular events on change in quality of life and utilities in patients after myocardial infarction. A VALIANT Study (Valsartan in acute myocardial infarction). JACC Heart Fail. 2014;2(2):159-65. https://doi.org/10.1 016/j.jchf.2013.12.003.

20. MacMahon S. Multimorbidity: a priority for global health research. London: The Academy of Medical Sciences; 2018.

21. Tisminetzky M, Goldberg R, Gurwitz JH. Magnitude and impact of multimorbidity on clinical outcomes in older adults with cardiovascular disease: a literature review. Clin Geriatr Med. 2016;32(2):227-46. https://doi. org/10.1016/j.cger.2016.01.014.

22. De Smedt D, Clays E, Annemans L, De Bacquer D, Doyle F, Kotseva K, et al Health related quality of life in coronary patients and its association with their cardiovascular risk profile: results from the EUROASPIRE III survey. Int Cardiol. 2013;168(2):898-903. https://doi.org/10.1016/j.ijcard.2012.10.053.

23. Pati S, Swain S, Knottnerus JA, Metsemakers JF, van den Akker M. Health related quality of life in multimorbidity: a primary-care based study from Odisha, India. Health Qual Life Outcomes. 2019;17(1):116. https://doi.org/1 0.1186/s12955-019-1180-3.

24. Alabas OA, West RM, Gillott RG, Khatib R, Hall AS, Gale CP, et al. Evaluation of the Methods and Management of Acute Coronary Events (EMMACE)-3: protocol for a longitudinal study. BMJ Open. 2015;5(6).

25. Thygesen K, Alpert JS, Jaffe AS, Simoons ML, Chaitman BR, White HD, et al. Third universal definition of myocardial infarction. Eur Heart J. 2012;33(20): 2551-67. https://doi.org/10.1093/eurheartj/ehs184.

26. Wilkinson C, Weston C, Timmis A, Quinn T, Keys A, Gale CP. The Myocardial Ischaemia National Audit Project (MINAP). Eur Heart J. 2020;6(1):19-22. https://doi.org/10.1093/ehjqcco/qcz052.
27. Brooks R, Group E. EuroQol: the current state of play. Health Policy. 1996; 37(1):53-72. https://doi.org/10.1016/0168-8510(96)00822-6.

28. Cheung K, Oemar M, Oppe M, Rabin R. EQ-5D User Guide. Basic information on how to use EQ-5D; 2009.

29. Nowels D, McGloin J, Westfall JM, Holcomb S. Validation of the EQ-5D quality of life instrument in patients after myocardial infarction. Qual Life Res. 2005;14(1):95-105. https://doi.org/10.1007/s11136-004-0614-4.

30. Nolan CM, Longworth L, Lord J, Canavan JL, Jones SE, Kon SS, et al. The EQ5D-5L health status questionnaire in COPD: validity, responsiveness and minimum important difference. Thorax. 2016:thoraxjnl-2015-207782.

31. Hagenaars JA, McCutcheon AL. Applied latent class analysis: Cambridge University Press; 2002.

32. Nylund-Gibson $\mathrm{K}$, Choi AY. Ten frequently asked questions about latent class analysis. Transl Issues Psychol Sci. 2018;4(4):440-61. https://doi.org/10.1 037/tps0000176

33. Everitt B, Landau S, Leese M, Stahl D. Cluster analysis; 2011. https://doi.org/1 0.1002/9780470977811.

34. Schwarz G. Estimating the dimension of a model. Ann Stat. 1978;6(2):461-4.

35. Konishi S, Kitagawa G. Information criteria and statistical modeling: Springer Science \& Business Media; 2008. https://doi.org/10.1007/978-0-387-71887-3.

36. Feng ZD, McCulloch CE. Using bootstrap likelihood ratios in finite mixture models. J R Stat Soc Ser B Methodol. 1996;58(3):609-17.

37. Patel RB, Colangelo LA, Reis JP, Lima JA, Shah SJ, Lloyd-Jones DM. Association of longitudinal trajectory of albuminuria in young adulthood with myocardial structure and function in later life: Coronary Artery Risk Development in Young Adults (CARDIA) study. JAMA Cardiol. 2020;5(2):18492. https://doi.org/10.1001/jamacardio.2019.4867.

38. Wu S, An S, Li W, Lichtenstein AH, Gao J, Kris-Etherton PM, et al. Association of trajectory of cardiovascular health score and incident cardiovascular disease. JAMA Netw Open. 2019;2(5):e194758-e.

39. Chen Y, Farooq S, Edwards J, Chew-Graham CA, Shiers D, Frisher M, et al. Patterns of symptoms before a diagnosis of first episode psychosis: a latent class analysis of UK primary care electronic health records. BMC Med. 2019; 17(1):1-13.

40. Steele F. Multilevel models for longitudinal data. J R Stat Soc Series A. 2008; 171(1):5-19.

41. Twisk J, Rijmen F. Longitudinal tobit regression: a new approach to analyze outcome variables with floor or ceiling effects. J Clin Epidemiol. 2009;62(9): 953-8. https://doi.org/10.1016/j.jclinepi.2008.10.003.

42. Yadegarfar ME, Gale CP, Dondo TB, Wilkinson CG, Cowie MR, Hall M. Association of treatments for acute myocardial infarction and survival for seven common comorbidity states: a nationwide cohort study. BMC Med. 2020;18(1):1-12.

43. Rutten-van Mölken MP, Oostenbrink JB, Tashkin DP, Burkhart D, Monz BU. Does quality of life of COPD patients as measured by the generic EuroQol five-dimension questionnaire differentiate between COPD severity stages? Chest. 2006;130(4):1117-28. https://doi.org/10.1378/chest.130.4.1117.

44. Eurich DT, Johnson JA, Reid KJ, Spertus JA. Assessing responsiveness of generic and specific health related quality of life measures in heart failure. Health Qual Life Outcomes. 2006;4(1):89. https://doi.org/10.1186/1477-752 5-4-89.

45. Szende A, Janssen B, Cabases J. Self-reported population health: an international perspective based on EQ-5D. Dordrecht: Springer Netherlands; 2014. https://doi.org/10.1007/978-94-007-7596-1.

46. Arifin $B$, Idrus LR, van Asselt AD, Purba FD, Perwitasari DA, Thobari JA, et al. Health-related quality of life in Indonesian type 2 diabetes mellitus outpatients measured with the Bahasa version of EQ-5D. Qual Life Res. 2019;28(5):1179-90.

47. Lu Y, Wang N, Chen Y, Nie X, Li Q, Han B, et al. Health-related quality of life in type-2 diabetes patients: a cross-sectional study in East China. BMC Endocr Disord. 2017;17(1):38.

48. Vaduganathan M, Fonarow GC, Greene SJ, DeVore AD, Albert NM, Duffy Cl, et al. Health-related quality of life in comorbid heart failure with reduced ejection fraction and diabetes mellitus. J Am Coll Cardiol. 2019;74(25):31768.

49. van Marwijk HW, van der Kooy KG, Stehouwer CD, Beekman AT, van Hout HPJ. Depression increases the onset of cardiovascular disease over and above other determinants in older primary care patients, a cohort study. BMC Cardiovasc Disord. 2015;15(1):40.

50. Birk JL, Kronish IM, Moise N, Falzon L, Yoon S, Davidson KWJHP. Depression and multimorbidity: considering temporal characteristics of the associations 
between depression and multiple chronic diseases. Health Psychol. 2019; 38(9):802.

51. Tromp J, Tay WT, Ouwerkerk W, Teng T-HK, Yap J, MacDonald MR, et al. Multimorbidity in patients with heart failure from 11 Asian regions: a prospective cohort study using the ASIAN-HF registry. PLoS Med. 2018;15(3): e1002541. https://doi.org/10.1371/journal.pmed.1002541.

52. Mori M, Krumholz HM, Allore HG. Using latent class analysis to identify hidden clinical phenotypes. Jama. 2020;324(7):700-1. https://doi.org/10.1 001/jama.2020.2278.

\section{Publisher's Note}

Springer Nature remains neutral with regard to jurisdictional claims in published maps and institutional affiliations.

Ready to submit your research? Choose BMC and benefit from:

- fast, convenient online submission

- thorough peer review by experienced researchers in your field

- rapid publication on acceptance

- support for research data, including large and complex data types

- gold Open Access which fosters wider collaboration and increased citations

- maximum visibility for your research: over $100 \mathrm{M}$ website views per year

At $\mathrm{BMC}$, research is always in progress.

Learn more biomedcentral.com/submissions 